\title{
REAL OPTIONS WITH UNKNOWN-DATE EVENTS ${ }^{\dagger}$
}

\author{
Óscar Gutiérrez Arnaiz \\ Department of Business Economics, \\ University of Zaragoza (Spain). \\ Francisco Ruiz-Aliseda \\ Kellogg School of Management, \\ Northwestern University (USA).
}

\begin{abstract}
The literature on real options has provided new insights on how to manage irreversible capital investments whose payoffs are always uncertain. Two of the most important predictions from the theory are: (i) greater risk delays investment timing by firms, and (ii) greater risk increases the option value of waiting. This paper shows that these conclusions need not be drawn from models in which the relevant random variable is the date of occurrence of an (unfavorable) event. We examine these issues in a setting in which a monopolist can launch a product whose profit follows an unknown temporal evolution.
\end{abstract}

Key words: Real Options, Investment under Uncertainty, Option Value, Entry Timing and Occurrence Date.

JEL Classification: D81, G31, L12.

\footnotetext{
† We would like to thank Vicente Salas-Fumás and Daniel Spulber for helpful comments that greatly improved the paper. Óscar Gutiérrez and Francisco Ruiz-Aliseda gratefully acknowledge financial support from Research Project MCYT-DGI/FEDER BEC2001-2552-C03-02 and Fundación la Caixa, respectively. The usual disclaimer applies.

E-mail: franruiz@kellogg.northwestern.edu
} 


\section{INTRODUCTION}

The literature on real options has largely modeled situations in which uncertainty regarding the value of a project makes it follow a continuous sample path. ${ }^{1}$ However, several investment decisions are mainly characterized by uncertainty referred to the occurrence date of a crucial event that would abruptly affect the value of the project.

For instance, consider the case of a firm that has to choose the timing of investment of an $R \& D$ project whose outcome can be patented. In winner-takes-all industries, the time until discovery of the product or technology is clearly one of the most critical factors to take into account because of competitive forces. Yet, the time-to-discovery is usually unknown ex ante, so it is reasonable to assume that the relevant randomness of such R\&D project largely stems from the uncertain discovery date, as well as those of competitors. ${ }^{2}$

As a second example, suppose that a company has to make a decision about when to build a factory subject to a probable change in environmental or tax policy. As discussed by Dixit and Pindyck (1994, p. 304), the date of policy change can be considered to be unknown to the firm and "it is commonly believed that expectations of shifts of policy can have powerful effects on decisions to invest".

Finally, let us consider product launching decisions. In particular, suppose that a firm has to decide when to introduce an already developed product under conditions of uncertainty about the future date at which a substitute product may be launched. The presence of this product may entail the gradual decline in the demand of the product sold by

\footnotetext{
${ }^{1}$ That is, the value of the project is usually assumed to follow a diffusion process, such as the geometric brownian motion. See, e.g., McDonald and Siegel (1986), Pindyck (1991) or Dixit and Pindyck (1994).

${ }^{2}$ This example is drawn from Weeds (2002), who formalizes such situations and characterizes the different equilibria that arise.
} 
the firm. Consequently, randomness about the maturity date of the market would be an aspect that would critically affect the firm's launching decision. ${ }^{3}$

The real options literature has certainly been aware of the importance of these situations in which the date of occurrence of a key event is uncertain. Indeed, this explains the use of Poisson processes in real option valuation. Yet, while Poisson arrivals seem the correct way to model such phenomena, this modeling approach is not particularly suited to perform many analyses beyond real option pricing. For example, the effects of meanpreserving spreads on investment timing and option values cannot be determined. For this reason, the purpose of this paper is to reexamine some of the conclusions of the theory of real options in a general setting in which all uncertainty refers to the instant of occurrence of an unfavorable event that critically affects the firm's payoff. ${ }^{4}$

The consideration of uncertain-date events yields that some of the fundamental results of the options approach are not clear-cut. In the first place, the canonical real options model predicts that the value of an investment opportunity is non-decreasing in the variance parameter of the geometric brownian motion that governs the return of the underlying asset. $^{5}$ Greater volatility cannot be harmful because the company always has the option to wait for better times or even not to invest should conditions turn out to be adverse. But, at the same time, the firm can capitalize on favorable market evolution and invest right on. Therefore, there exists an asymmetry due to the following: on the one hand, the firm is insured against adverse realizations of uncertainty by waiting; on the other, it can simultaneously take advantage of beneficial events. This asymmetry, always present when

\footnotetext{
${ }^{3}$ Such situation is modeled for example in Bollen (1999).

${ }^{4}$ Using the term coined by Samuel-Cahn (1996), the date of occurrence of such event is a random "freezetime" variable. One could also think of it as a non-homogeneous Poisson process with a time-dependent arrival date in which only the first event that occurs (and its associated effect on the firm's value) matters.

${ }^{5}$ See, e.g., the classic paper by McDonald and Siegel (1986).
} 
there exist options whose payoffs are convex in the random variable, accounts for the nonnegative effects of volatility on the value of investment opportunities. Notwithstanding, one of the contributions of our paper is to show that such asymmetry is not present when there exists uncertainty about the date of the unfavorable event. In principle, the event may occur before or after investing, but in equilibrium, it can be shown that the firm finds it optimal to undertake only the investment if its payoff is uncertain. Given this fact and that the space of outcomes coincides with that of time, we have that the firm would be insured against bad realizations because of waiting, and would take advantage of good ones, but it would be damaged by realizations sufficiently close to the optimal time of investment. That is, if the event occurred right after investing, then the project may turn out to be unprofitable ex post, even though it might have seemed an excellent investment ex ante. As a result, more uncertainty may destroy option values, depending on whether a mean-preserving spread increases sufficiently the probability of occurrence of the event once investment has taken place.

In the second place, real options theory usually predicts that increased risk delays investment timing, a relevant aspect for both public policy and business purposes. Such conclusion basically follows from the "bad news principle of irreversible investment" (see Bernanke (1983)). The intuition behind such principle is that the benefit from waiting arises from the avoidance of making a poor investment when news is bad (i.e., when events turn out to be unfavorable). Given that only adverse events matter and a mean-preserving spread increases their probability of occurrence, the marginal benefit from waiting increases with uncertainty. On the other hand, the marginal opportunity cost of waiting (namely, current profits foregone) is not affected by the spread. Consequently, the net marginal benefit of waiting increases with uncertainty, which in turn induces a delay in entry. We show that 
this need not be true in our setting. More specifically, in our model only adverse events matter too. Yet, the firm endogenously chooses whether to position itself in a situation in which a mean-preserving spread increases or decreases the probability of occurrence of the event, which explains why the conclusions may differ.

It is not unusual to find the claim that real options theory predicts that greater volatility depresses investment, and at the same time increases the value of a firm's investment opportunities. These conclusions can already be found in McDonald and Siegel (1986, p. 714). Indeed, the prediction that more uncertainty leads to less investment appears to be supported by empirical evidence, as shown by Ferderer (1993) using aggregate data or Leahy and Whited (1996) and Guiso and Parigi (1999) using micro data. These two last studies suggest that real options theory is the most solid theory of investment under uncertainty, which reinforces the need for a more comprehensive framework that helps to determine the factors and conditions that drive theoretical conclusions. Our work tries to be a step in this direction.

The remainder of the paper is organized as follows. Section 2 introduces the model we analyze, as well as its assumptions, whereas Section 3 solves it. Section 4 identifies a necessary and sufficient condition under which an increase in risk speeds up investment and provides a numerical illustration. In turn, Section 5 provides a necessary and sufficient condition for the value of an investment opportunity to be a non-decreasing function of volatility, while Section 6 concludes. A mathematical appendix with all proofs is included at the end of the paper. 


\section{FOUNDATIONS OF THE THEORETICAL MODEL}

Let time, denoted by $t$, be a continuous variable, i.e. $t \in[0, \infty)$. Suppose that a risk-neutral monopolist has to make a decision about the instant at which it wants to launch a product for which there already exist some potential buyers. Such decision is complicated by the existence of uncertainty about the temporal evolution of the market, which in turn affects the pattern of profit evolution. Uncertainty is assumed to unravel partially over time and the market (and, as a result, profit) is assumed to evolve in the following manner. In a first stage, the profit flow, which is positive at date $t=0$, grows over time. However, the market reaches its ephemeral maturity at an instant of time $\tau$, where $\tau$ is a continuous random variable with known density $f(\tau)$ defined on $(0, \infty)$. (We will slightly abuse the notation and $\tau$ will also denote its realization.) Hence, in a second phase whose beginning is unknown at date 0 , instantaneous profit decreases over time and converges to 0 as $t \rightarrow \infty$, perhaps because consumers perceive that there is another product that can serve their needs better and choose to switch.

Formally, we make the following assumptions:

Assumption 1: Instantaneous profit made by the monopolist if active in the market evolves over time as follows:

$$
\Pi(t, \tau)= \begin{cases}\pi \exp (\alpha t) & \text { if } 0 \leq t \leq \tau \\ \pi \exp [\alpha(2 \tau-t)] & \text { if } t>\tau\end{cases}
$$

$\pi$ denotes the profit made by the monopolist at date $t=0$ and is assumed to be positive, ${ }^{6}$ while $\alpha>0$ denotes the growth rate of the profit flow (if the market is in expansion; otherwise, it is its decay rate). ${ }^{7}$

\footnotetext{
${ }^{6} \mathrm{We}$ are implicitly assuming that either operating costs are small enough or they are zero, in order to avoid making exit an issue and focus only on entry timing. Zero cost is not necessary, though. For example, in a
} 
Assumption 2: The maturity date of the market $\tau$ is a random variable with continuous density function $f(\tau)$ with support $(0, \infty)$.

In order to set up a real options framework, we require investment to be irreversible:

Assumption 3: The monopolist bears an entirely sunk cost of entry $K>0$ and discounts future payoffs at a constant risk-free interest rate $r \geq 0$.

Henceforward, denote the expectation operator conditional upon information at time $t$ by $E_{t}(\cdot)$ (the expectation is taken over $\tau$ ). Assumption 4 below ensures that the expected discounted value of one dollar that is capitalized at an instantaneous rate of $\alpha$ is finite no matter what the duration of the ascending phase of the profit cycle is. This bounds the value of the monopolist's investment opportunity. ${ }^{8}$

Assumption 4: $E_{t}\left(e^{(\alpha-r) \tau}\right)<\infty \forall t \geq 0$.

\section{RESOLUTION OF THE MODEL}

The firm's objective at time $t=0$ is to choose an entry rule that maximizes the expected discounted stream of cash flows conditional upon information available at the time of entry. We proceed now to characterize such optimal entry rule for the two possible states of the system, depending on whether the maturity date of the market has been revealed or not.

In the first place, it is clear that it is not optimal for the firm to exit at some date after having invested, given our assumptions of no scrap value and positive instantaneous profit

\footnotetext{
linear city model such as Hotelling's, a single firm located at one extreme always makes profits as long as $c<v-t$, where $c$ is the constant marginal cost of production, $v$ is the consumers' valuation of the good, and $t$ is the transport cost per unit of distance traveled incurred by buyers. If the measure of consumers $A$ is uniformly distributed along the line, then profits would always be positive: $\pi=A(v-c-t)>0$. Hence, exit would never occur in this model, since costs are always transferred to consumers, who can afford it.

${ }^{7}$ It is straightforward to introduce different rates of growth and decay. We choose not to do so to keep the model simple.

${ }^{8}$ In the traditional real options framework, a parallel convergence condition requires the rate of expected growth of the investment to be smaller than the risk-free rate.
} 
at any possible situation. This holds no matter if $\tau$ is known or not. In turn, Lemma 1 below describes the firm's behavior once the maturity of the market has been reached. According to this result, the firm prefers to invest immediately once $\tau$ is revealed, but only if such date is sufficiently large; otherwise, it prefers not to invest:

Lemma 1: Immediate investment at the revealed maturity date $\tau$ is optimal $\forall \tau \geq t^{\max }=\max \left[0, \frac{1}{\alpha} \log \left(\frac{K(\alpha+r)}{\pi}\right)\right]$, whereas investment during the declining phase of the market is not profitable $\forall \tau<t^{\max }$.

Hence, to characterize the optimal entry rule fully, it only remains to focus on the time $t_{1}$ at which the firm would enter the market if $\tau$ had not been revealed yet, and thus the profit cycle were in its ascending phase. Using Lemma 1, the value of the monopolist's investment opportunity at date $t=0$ as a function of its entry time $t_{1} \geq 0$ is:

$$
V\left(t_{1}\right)=\int_{0}^{t_{1}} f(\tau) \max \left(0, \int_{\tau}^{\infty} \Pi(s, \tau) e^{-r s} d s-K e^{-r \tau}\right) d \tau+\int_{t_{1}}^{\infty} f(\tau)\left(\int_{t_{1}}^{\infty} \Pi(s, \tau) e^{-r s} d s-K e^{-r t_{1}}\right) d \tau
$$

If the firm chooses to wait until $t_{1}$, then, for realizations smaller than $t_{1}$, it seizes the payoff to immediate investment at $\tau$ if and only if it is non-negative. In contrast, if the firm ends up entering at $t_{1}$ while the profit cycle is growing, then it seizes an expected payoff that is conditional upon the information at time $t_{1}$ (namely, that $\tau$ must be greater than $t_{1}$ ). Note that, by another application of the lemma, $V\left(t_{1}\right)$ can be rewritten as follows:

$$
V\left(t_{1}\right)=\left\{\begin{array}{lr}
\int_{t_{1}}^{\infty} f(\tau)\left(\int_{t_{1}}^{\infty} \Pi(s, \tau) e^{-r s} d s-K e^{-r t_{1}}\right) d \tau & \text { if } t_{1} \in\left[0, t^{\max }\right) \\
\int_{t_{\max }}^{t_{1}} f(\tau)\left(\int_{\tau}^{\infty} \Pi(s, \tau) e^{-r s} d s-K e^{-r \tau}\right) d \tau+\int_{t_{1}}^{\infty} f(\tau)\left(\int_{t_{1}}^{\infty} \Pi(s, \tau) e^{-r s} d s-K e^{-r t_{1}}\right) d \tau & \text { else }
\end{array}\right.
$$


$V\left(t_{1}\right)$ can be easily shown to be continuously differentiable. So, unlike conventional real option analysis, in which expected payoff functions depend on Ito processes and thereby are not differentiable in the classical sense, the monopolist's optimization program can be solved using standard differentiation techniques:

$$
\begin{aligned}
& \max _{t_{1} \geq 0} V\left(t_{1}\right) \\
& \text { s.t. } \int_{t_{1}}^{\infty} f(\tau)\left(\int_{t_{1}}^{\infty} \Pi(s, \tau) e^{-r s} d s-K e^{-r t_{1}}\right) d \tau \geq 0
\end{aligned}
$$

The non-negativity constraint requires the firm to make a profitable investment, in expectation, when the market is still growing. It is clear that the ex post non-negativity constraint we should have included is directly satisfied. However, Lemma 1 has additional implications for the ex ante constraint, since letting $t_{1}=t^{\max }$ would automatically satisfy the constraint. In other words, the lemma implies that there exists an instant of time $t^{\text {max }}$ such that, if the market kept on growing, profits would be so large that the firm would find it profitable to enter the industry even though the market began to decay right after entry. Hence, the value of the option to invest when the cycle is growing can never be negative at the optimum, and thus the constraint can be dropped from the firm's optimization program.

Now we can examine the firm's optimal decision rule, but let us first introduce some notation. In particular, let $\lambda(t)=\frac{f(t)}{\int_{t}^{\infty} f(\tau) d \tau}$ denote the hazard rate, that is, the instantaneous probability of the market reaching its maturity at date $t$ given that this event has not occurred previously. Let us assume that it is a differentiable function, with $\frac{d \lambda(t)}{d t} \leq 0$ to 
ensure that $V(\cdot)$ is single-peaked..$^{9}$ Thus, we can characterize the firm's optimal rule of entry:

Lemma 2: The monopolist's optimal entry rule is "enter at $t^{*}=t^{e}$ if $t^{e} \leq \tau$; else do not enter" if there exists a unique $t^{e} \in\left(0, t^{\max }\right)$ such that

$$
e^{\alpha t^{e}}\left(1+\frac{\alpha}{r+\lambda\left(t^{e}\right)}\right)=\frac{K(\alpha+r)}{\pi} .
$$

The monopolist's optimal rule is "enter at $t^{*}=0$ " if such $t^{e}$ does not exist.

We can draw a corollary from Lemma 2 that allows us to give an economic interpretation to the firm's optimization problem:

Corollary 1: If $\tau$ has not been realized yet and the optimal entry time $t^{e}$ exists, then it is such that:

$$
\pi e^{\alpha t^{e}} d t=r K d t+\lambda\left(t^{e}\right)\left(K-\int_{t^{e}}^{\infty} \pi e^{\alpha\left(2 t^{e}-s\right)} e^{-r\left(s-t^{e}\right)} d s\right) d t .
$$

The corollary means that if the profit cycle is still growing and the solution to the program is interior, then the monopolist decides to enter at the instant of time such that the marginal value of waiting equals the marginal cost of delaying entry. The marginal cost is the instantaneous profit foregone by waiting $d t: \pi e^{a t e} d t$. In turn, marginal value is sunk cost saved by delaying entry plus the marginal option value of waiting and avoiding an irreversible action. The latter value stems from the "bad news principle of irreversible investments" that can be found in Bernanke (1983). This principle indicates that the firm

\footnotetext{
${ }^{9}$ Log-convexity of the density function implies non-increasing hazard rate. Familiar probability distributions with log-convex density and support $(0, \infty)$ include the exponential, gamma and Weibull (in the latter two cases if the shape parameter is no larger than 1) and the F-distribution with the first degree of freedom no larger than 2. This condition could be relaxed to allow for distributions with an increasing hazard rate for
} 
only cares about the bad news that may arrive in the next instant of time when deciding whether or not to undertake an irreversible project. ${ }^{10}$ Thus, the firm believes that the demand of the product may suddenly decay right after time $t^{e}$ (this is the bad news), so by waiting the firm believes it would elude making a negative payoff with probability $\lambda\left(t^{e}\right) d t .{ }^{11}$ Overall, total marginal value is equal to:

$$
r K d t+\lambda\left(t^{e}\right)\left(K-\int_{t^{e}}^{\infty} \pi e^{\alpha\left(2 t^{e}-s\right)} e^{-r\left(s-t^{e}\right)} d s\right) d t .
$$

Note from the second term that, in general, waiting allows the firm to update its beliefs about the time at which maturity is reached. ${ }^{12}$ By waiting, the firm benefits from learning what some events cannot be (via the denominator of the hazard rate; see its definition), which in principle allows it to have a better assessment of the probabilities of still-notoccurred-events. ${ }^{13}$ At the same time, waiting implies that the firm faces a smaller probability of the market immediately reaching its maturity (via the numerator of the hazard rate)..$^{14}$

We can thus conclude with the characterization of the solution by analyzing how the value of the firm's investment opportunity depends on initial profit. $t^{*}=0$ if and only if $t^{e}$

some ranges, such as the lognormal distribution. In particular, letting $\alpha>\sup \frac{d \lambda(t) / d t}{\lambda(t)}$ would suffice. It is worthwhile mentioning that our results would continue to hold under such assumption.

${ }^{10}$ The intuition is that irreversibility yields no advantages but implies some costs that arise because the firm cannot recoup its investment if conditions turn out to be adverse. Thus, irreversibility creates the asymmetry that the firm cares about adverse events (which would not be regrettable were investment reversible) but not favorable ones.

${ }^{11}$ This follows from Lemma 1, which implies that the payoff to the firm if it invested at date $t$ is negative if demand suddenly decays $\forall t<t^{\max }$.

${ }^{12}$ If the stochastic peak is assumed to follow an exponential distribution, pursuing a business strategy of "wait and see" does not allow for an updating of information via hazard rate, since the latter is invariant to time (this is due to the so called lack-of-memory property).

${ }^{13}$ The reason being that conditioning reduces the outcome space.

${ }^{14}$ Note that a necessary condition for a non-increasing hazard rate is a decreasing density, which explains the result. 
does not exist, so that $t^{*}=0$ if and only if $\pi \geq \pi^{*}=\frac{K(\alpha+r)(r+f(0))}{\alpha+r+f(0)}$, given that $\lim _{t \downarrow 0} \lambda(t)=\frac{\lim _{t \downarrow 0} f(t)}{\lim _{t \downarrow 0} \int_{t}^{\infty} f(\tau) d \tau}=\lim _{t \downarrow 0} f(t) \equiv f(0)$. (Yet, note that $\lim _{t \downarrow 0} \lambda(t)=\lambda$ if and only if the probability distribution is exponential with parameter $\lambda$.) Taking this into account, the investment opportunity as a function of date 0 's profit $\pi$ is:

$$
V(\pi)= \begin{cases}\pi\left(\frac{1}{\alpha+r}+\frac{1}{\alpha-r}\right) \int_{t^{e}}^{\infty} e^{(\alpha-r) \tau} f(\tau) d \tau-\left(\frac{\pi e^{(\alpha-r) t^{e}}}{\alpha-r}+K e^{-r t^{e}}\right) \int_{t^{e}}^{\infty} f(\tau) d \tau & \text { if } \pi<\pi^{*} \\ \pi\left(\frac{1}{\alpha+r}+\frac{1}{\alpha-r}\right) \int_{0}^{\infty} e^{(\alpha-r) \tau} f(\tau) d \tau-\left(\frac{\pi}{\alpha-r}+K\right) & \text { else }\end{cases}
$$

\section{IMPACT OF GREATER UNCERTAINTY ON ENTRY TIMING}

One of the standard predictions of real options models is that more uncertainty delays the optimal time of investment. There are some exceptions, though. Dixit and Pindyck (1994) observe that greater uncertainty may actually hasten investment if the instantaneous profit function is convex in the variable that follows a stochastic process (e.g., price). Then, by Jensen's inequality, a larger variance implies a greater expected present value of the profit stream, thus increasing incentives to invest earlier.

Bar-Ilan and Strange (1996) also conclude that uncertainty may speed up investment in a model with investment lags and option to abandon. The reason is that, although the benefit of not undertaking an irreversible investment increases with uncertainty, the opportunity cost of waiting is also increasing with uncertainty. Waiting implies that the firm makes no profits for a certain period of time, so profits are uncertain as they have to be realized in the future due to the existence of investment lags. Since the existence of the 
option to abandon bounds below the profits that the firm can make in the future, an increase in uncertainty increases expected profits of waiting again by Jensen's inequality. Therefore, the increase in the opportunity cost of waiting may be larger than the benefit of waiting, thus speeding up investment.

We now show that the effect on investment timing of a greater spread is also ambiguous when the payoff to the firm crucially depends on the unknown date of occurrence of an unfavorable event. In particular, we give a necessary and sufficient condition for a mean-preserving spread to shorten the optimal time of entry. For this reason, we make the assumption that $\pi<\pi^{*}$, so $t^{*}=t^{e}>0$. Also, we assume throughout this section and the following one that the density can be parameterized by $\sigma^{2} \in \mathfrak{R}_{+}$, i.e. $f\left(\tau \mid \sigma^{2}\right) . \sigma^{2}$ is a parameter such that a rise in it represents a mean-preserving spread, so we will usually interpret as the variance of the random variable. As a result, the hazard rate is also a function of $\sigma^{2}, \lambda\left(t \mid \sigma^{2}\right)$, and, for convenience, we assume that it is differentiable in both arguments. Denoting partial derivatives by subscripts, we recall the assumption we made in the previous section: $\lambda_{t}\left(t \mid \sigma^{2}\right) \leq 0$. Lastly, let us assume for the remainder of the paper that $G\left(t^{e}, \sigma^{2}\right)=e^{\alpha t^{e}}\left(1+\frac{\alpha}{r+\lambda\left(t^{e} \mid \sigma^{2}\right)}\right)-\frac{K(\alpha+r)}{\pi}$ is a continuously differentiable function on the neighborhood of any pair $\left(t_{0}^{e}, \sigma_{0}^{2}\right)$ such that $G\left(t_{0}^{e}, \sigma_{0}^{2}\right)=0$. Then we can establish the following:

Proposition 1: A mean-preserving spread hastens investment if and only if $\lambda_{\sigma^{2}}\left(t_{0}^{e} \mid \sigma_{0}^{2}\right)<0$.

Assuming for expositional purposes that $f(\tau \mid \cdot)$ is a continuously differentiable function $\forall \tau$, it follows that $\lambda_{\sigma^{2}}\left(t_{0}^{e} \mid \sigma_{0}^{2}\right)<0$ if and only 


$$
f_{\sigma^{2}}\left(t_{0}^{e} \mid \sigma_{0}^{2}\right)+\lambda\left(t_{0}^{e} \mid \sigma_{0}^{2}\right) \int_{0}^{t_{0}^{e}} f_{\sigma^{2}}\left(\tau \mid \sigma_{0}^{2}\right) d \tau<0
$$

Hence, a mean-preserving spread has two distinct effects that influence optimal investment timing. On the one hand, it has an effect on the probability of immediate decay $f\left(t_{0}^{e} \mid \sigma_{0}^{2}\right)$. On the other, there is an additional effect on the value of knowledge attributable to waiting until $t_{0}^{e}, \int_{0}^{t_{0}^{e}} f\left(\tau \mid \sigma_{0}^{2}\right) d \tau$. Thus, in the most interesting cases in which both effects are in conflict, investment is hastened if:

- Either the better assessment of future events is relatively less important than the decrease in the probability of abrupt decay (a sort of "good news principle").

- Or the probability of immediate failure increases little relative to the decrease in the value of accumulated knowledge. This implies that the assessment of the instantaneous probabilities of future decay worsens, which blurs the belief that irreversibility is costly for the firm.

Either way, the marginal option value of waiting (i.e., the cost of irreversibility) would diminish due to the decrease on the weight put on the losses avoided by delaying entry. However, the effect of uncertainty on the value of (probabilistic) knowledge is absent in the conventional real options literature. This is due to our setting in which the firm can learn about the unknown date of occurrence of the maturity date by waiting, which allows it to enhance its information set. Yet, our model predicts that entry may be hastened by a greater spread, even though the value of knowledge were unaffected, as long as $f\left(t_{0}^{e} \mid \sigma_{0}^{2}\right)$ decreases. 
Let us next give a numerical illustration of Proposition 1. In particular, assume that $\tau$ follows a gamma distribution with parameters $\gamma>0$ and $\rho>0: \tau \sim G(\gamma, \rho)$. Recall that $E(\tau)=\frac{\rho}{\gamma}$ and $\operatorname{Var}(\tau)=\frac{\rho}{\gamma^{2}}$, which means that we can perform mean-preserving spreads by simply multiplying both $\gamma$ and $\rho$ by any positive scalar smaller than 1 . In addition, let the monopolist face a linear demand with intercept $a=50$ and slope $b=1$. If costs are assumed to be zero, then it is well-known that $\pi=\frac{50^{2}}{4}$. Finally, let $K=7000$ and $r=\alpha=5 \%$. Considering that the hazard rate of the gamma is non-increasing if and only if $\rho \leq 1$, then we have the following results, summarized in Table 1:

\begin{tabular}{|c|c|c|c|c|}
\hline$\rho$ & $\gamma$ & $E(\tau)$ & $\operatorname{Var}(\tau)$ & $t^{*}=t^{e}$ \\
\hline 0.8 & 0.4 & 2 & 5 & 0.58 \\
\hline 0.5 & 0.25 & 2 & 8 & 0.56 \\
\hline 0.1 & 0.05 & 2 & 40 & 0.2 \\
\hline
\end{tabular}

Table 1: More uncertainty speeds up entry

\section{IMPACT OF GREATER UNCERTAINTY ON OPTION VALUES}

Traditional real option theory predicts that an increase in volatility does not harm the value of the monopolist's investment opportunity (see Dixit and Pindyck (1994) or Trigeorgis (1996)). We next study conditions under which this may not happen in our setting. More precisely, we perform a comparative static analysis on the impact of a mean-preserving spread on the value of the investment opportunity, which requires additional notation. In particular, let $F\left(\tau \mid \sigma^{2}\right)$ denote the cumulative distribution function of $\tau$. 
Let us first modify some results from Section 3 to take into account the fact that we are to perform a comparative statics analysis on $\sigma^{2}$. Thus, the threshold that optimally triggers immediate investment by the firm becomes a function of $\sigma^{2}$ :

$$
\pi^{*}\left(\sigma^{2}\right)=\frac{K(\alpha+r)\left(r+f\left(0 \mid \sigma^{2}\right)\right)}{\alpha+r+f\left(0 \mid \sigma^{2}\right)} .
$$

In addition, the value of the investment opportunity as a function of both $\pi$ and $\sigma^{2}$ becomes:

$$
V\left(\pi, \sigma^{2}\right)= \begin{cases}\pi\left(\frac{1}{\alpha+r}+\frac{1}{\alpha-r}\right) \int_{t^{e}\left(\sigma^{2}\right)}^{\infty} e^{(\alpha-r) \tau} f\left(\tau \mid \sigma^{2}\right) d \tau- & \\ \left(\frac{\pi e^{(\alpha-r) t^{e}\left(\sigma^{2}\right)}}{\alpha-r}+K e^{-r t^{e}\left(\sigma^{2}\right)}\right) \int_{t^{e}\left(\sigma^{2}\right)}^{\infty} f\left(\tau \mid \sigma^{2}\right) d \tau & \text { if } \pi \geq \pi^{*}\left(\sigma^{2}\right) \\ \pi\left(\frac{1}{\alpha+r}+\frac{1}{\alpha-r}\right) \int_{0}^{\infty} e^{(\alpha-r) \tau} f\left(\tau \mid \sigma^{2}\right) d \tau-\left(\frac{\pi}{\alpha-r}+K\right) & \text { else }\end{cases}
$$

As readily seen from this expression, comparative statics are slightly complicated because the value of the investment opportunity is a piecewise differentiable function of $\pi$ and the non-differentiability point $\pi^{*}$ depends on $\sigma^{2}$. For this reason, we make a mild assumption that can be relaxed for some specific probability distributions that do not satisfy it. In particular, we assume that $f\left(0 \mid \sigma^{2}\right)$ is a continuous function. ${ }^{15}$ Now we can characterize some relevant properties of $\pi^{*}\left(\sigma^{2}\right)$ :

Lemma 3: $\pi^{*}\left(\sigma^{2}\right)$ is a continuous and non-decreasing function with range bounded by the interval $[r K,(\alpha+r) K]$.

Lemma 3 states that $\pi^{*}\left(\sigma^{2}\right)$ is non-decreasing in $\sigma^{2}$, so a mean-preserving spread usually makes investment at $t=0$ more difficult as happens in traditional real options

\footnotetext{
${ }^{15}$ For example, the lognormal distribution satisfies such assumption, since $f\left(0 \mid \sigma^{2}\right)=0 \forall \sigma^{2}$.
} 
models. Yet, note that, as Proposition 1 shows, investment timing need not be delayed, because the firm may not wish to invest at $t=0$ even for a low variance. We can now establish a necessary and sufficient condition under which a mean-preserving spread does not reduce the value of the investment opportunity:

Proposition 2: A mean-preserving spread does not reduce the value of the investment opportunity if and only if $2\left(\alpha+\lambda\left(t_{0}^{*} \mid \sigma_{0}^{2}\right)\right) \int_{t_{0}^{*}}^{\infty} e^{(\alpha-r)\left(\tau-t_{0}^{*}\right)} F_{\sigma^{2}}\left(\tau \mid \sigma_{0}^{2}\right) d \tau \leq \int_{0}^{t_{0}^{*}} f_{\sigma^{2}}\left(\tau \mid \sigma_{0}^{2}\right) d \tau$.

The proof of the proposition shows that increasing the risk of the investment opportunity has two effects. To identify them, let $t_{0}^{*}=t_{0}^{e}>0$ as well as $\alpha=r$ (so as to eliminate an additional effect due to the existence of discounting) ${ }^{16}$ and note that, by expression (A2) in the Appendix, the following holds:

$$
V^{\prime}\left(\sigma_{0}^{2}\right)=\left(\frac{\pi}{2 \alpha}-K e^{-\alpha t_{0}^{e}}\right)_{t_{0}^{e}}^{\infty} f_{\sigma^{2}}\left(\tau \mid \sigma_{0}^{2}\right) d \tau-\pi \int_{t_{0}^{e}}^{\infty} F_{\sigma^{2}}\left(\tau \mid \sigma_{0}^{2}\right) d \tau
$$

This can be rewritten as:

$$
V^{\prime}\left(\sigma_{0}^{2}\right)=\left(\frac{\pi}{2 \alpha}-K e^{-\alpha t_{0}^{e}}\right) \int_{t_{0}^{e}}^{\infty} f_{\sigma^{2}}\left(\tau \mid \sigma_{0}^{2}\right) d \tau+\pi t_{0}^{e}\left(\frac{\int_{0}^{t_{0}^{e}} F_{\sigma^{2}}\left(\tau \mid \sigma_{0}^{2}\right) d \tau}{t_{0}^{e}}\right),
$$

\footnotetext{
${ }^{16}$ As discussed by Klette and de Meza (1986) when performing a mean-preserving spread given a symmetric density, the existence of a discount rate (in our case, net of $\alpha$, the capitalization rate) has the general implication that delaying the receipt of a sum of money by $t$ years does not decrease the present value by the same amount as an advance of $t$ years increases it. When $\alpha=r$, this no longer holds and the decrease equals the increase. Furthermore, this allows us to compare delays of $t$ years with advances of $s$ years, where $s \neq t$, so the symmetric density assumption is also irrelevant.
} 
since $t_{0}^{e}>0$ and $\int_{0}^{\infty} F_{\sigma^{2}}\left(\tau \mid \sigma_{0}^{2}\right) d \tau=0$ when a mean-preserving spread is performed. ${ }^{17}$ The two effects of higher risk on the value of the investment opportunity can be seen from this expression.

On the one hand, the first term is absent in conventional real option models. It represents the effect on the firm's payoff of increased risk on the probability of occurrence of the event after investing, since $\frac{\pi}{2 \alpha}-K e^{-\alpha t_{0}^{e}}$ is the maximum loss made by the firm, ${ }^{18}$ which occurs if the market reaches its maturity right after investing at $t_{0}^{e}$. On the other, the second term in the expression is non-negative $\forall t_{0}^{e}{ }^{19}$ and represents the "option value" effect: the decrease in the (maximum) expected opportunity cost of waiting until $t_{0}^{e}>0$.

The reason is that $\frac{\int_{0}^{t_{0}^{e}} F_{\sigma^{2}}\left(\tau \mid \sigma_{0}^{2}\right) d \tau}{t_{0}^{e}}>0$ is the average increase in the probability of decay of the cycle before it reaches $t_{0}^{e}$, whereas $\pi t_{0}^{e}>0$ is the maximum amount of profits foregone by delaying investment until $t_{0}^{e} .^{20}$

Overall, the sum of both effects can be positive or negative, as can be readily seen from the necessary and sufficient condition provided in Proposition 2, $2\left(\alpha+\lambda\left(t_{0}^{e} \mid \sigma_{0}^{2}\right)\right) \int_{t_{0}^{e}}^{\infty} F_{\sigma^{2}}\left(\tau \mid \sigma_{0}^{2}\right) d \tau \leq \int_{0}^{t_{0}^{e}} f_{\sigma^{2}}\left(\tau \mid \sigma_{0}^{2}\right) d \tau$, given that the left hand side is always nonpositive and the right hand side has an undefined sign. We next provide an example in

\footnotetext{
${ }^{17}$ See Tirole (1988, p. 397, exercise 10.6), or Mas-Colell, Whinston and Green (1995, p. 198, expression 6.D.1) for an informal argument.

${ }^{18}$ Note that $\frac{\pi}{2 \alpha}<K e^{-\alpha t_{0}^{e}}$ by the first-order condition of the program solved by the entrant.
} 
which volatility harms option values. Let us continue with the example of the previous section. Noting that the gamma distribution has $f\left(0 \mid \sigma^{2}\right)=\infty \forall \sigma^{2}$ if and only if its hazard rate is decreasing, we have the results depicted in Table 2.

\begin{tabular}{|c|c|c|c|c|}
\hline$\rho$ & $\gamma$ & $E(\tau)$ & $\operatorname{Var}(\tau)$ & $V$ \\
\hline 0.8 & 0.4 & 2 & 5 & 560 \\
\hline 0.5 & 0.25 & 2 & 8 & 627 \\
\hline 0.1 & 0.05 & 2 & 40 & 598 \\
\hline
\end{tabular}

Table 2: More uncertainty harms option values

Thus, the usual asymmetry originated by the fact that the firm can take advantage of upside risk without being affected by downside risk is not present in this situation any more. The ex post value of the investment opportunity is not convex in the realization of the random variable unless $t^{*}=0$. Indeed, it is not a continuous function of $\tau$, as illustrated by Figure 1. It is worth remarking that this result is very specific to settings similar to ours. The critical reason is that, in these cases, the outcome space coincides with that of $t$, which allows the discontinuity to appear. Thus, the firm does not invest when its worst-scenario payoff is 0 (the only way to prevent the discontinuity from arising), but rather when its expected payoff is maximized, that is, at $t^{e}$. Yet, the monopolist faces risk of losses for realizations sufficiently close to $t^{e}$ (since $t^{e}<t^{\max }$ ), as readily seen in Figure 1(a). So it is this risk of losses that explains why increasing the spread may partially destroy option values.

\footnotetext{
${ }^{19}$ See Tirole (1988, p. 397, exercise 10.6) or Mas-Colell, Whinston and Green (1995, p. 198, expressions 6.D.1 and 6.D.2).
} 
(a)Present paper framework

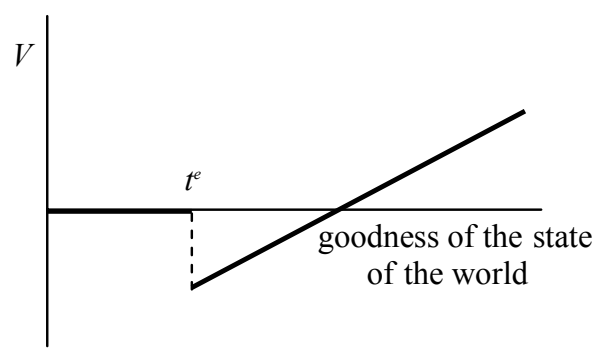

(b)Traditional framework

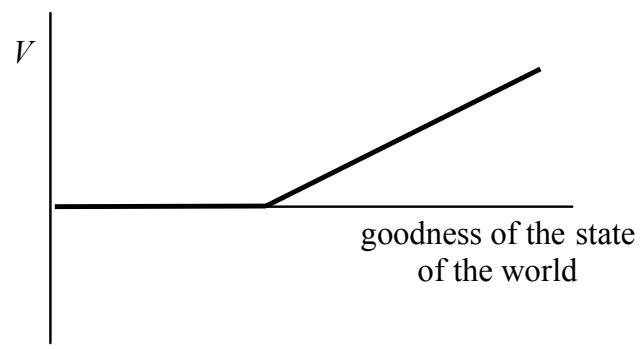

Figure 1: Ex post value of the investment opportunity

As a result, the firm is insured against bad realizations because of waiting, and definitely takes advantage of good realizations, but it is damaged by realizations sufficiently close to $t^{e}$. The overall effect of greater uncertainty is thus ambiguous.

\section{CONCLUSION}

This paper has focused on investment contexts in which the only source of uncertainty affecting the value of a project stems from the unknown date of occurrence of an unfavorable event. We have departed from modeling uncertainty over time through a stochastic process, and we have assumed under fairly general conditions that the date of occurrence of the event is a random variable, as opposed to a Poisson process, which is not well suited for certain analyses.

The consideration of unknown-date events as a source of uncertainty dramatically affects some of the core conclusions that characterize conventional real options theory. More specifically, we have identified a necessary and sufficient condition under which the value of an investment opportunity decreases with risk. The motive why this may occur is that the time space and the outcome space coincide. Consequently, waiting allows the firm

\footnotetext{
${ }^{20}$ The discount rate does not appear because recall that we have assumed $\alpha=r$ for expositional purposes.
} 
to be insured against adverse states of the world, while taking advantage of favorable states. However, the firm cannot avoid being damaged and thus making losses for realizations sufficiently close to the optimal time of investment. The reason is that the firm decides to invest when expected net present value is maximal, not when downside risk disappears, and thus an increase in risk may increase the probability of occurrence of the adverse event by a sufficiently large amount.

In addition, we have shown that increased risk may speed up entry timing in certain situations. Pinpointing the conditions under which this occurs is certainly relevant for empirical work on the investment-uncertainty relationship. Our model shows that, at the margin, the firm cares about the probability of immediate occurrence of the unfavorable event. An increase in uncertainty may reduce this probability, thus decreasing the cost of making an irreversible investment, which would hasten entry. Greater uncertainty has an additional effect also to be taken into account: the impact on the value of information about the occurrence of the event in the future, ${ }^{21}$ an aspect missing on conventional models.

Lastly, we have set up an alternative theoretical framework for continuous-time real option models of investment whenever the demand of a product follows a life cycle that is unknown to the firm. ${ }^{22}$ The setting is simpler than that proposed by Bollen (1998) and is a potentially useful building block for issues that we do not deal with, such as the option to

\footnotetext{
${ }^{21}$ This contrasts with Roberts' and Weitzman's (1981) model of staged investment, in which the value of a project is also unknown to the company but the firm can reduce uncertainty by going ahead in a sequential fashion. Yet, in our stylized model, unlike theirs, information gathering does not require an earlier investment. Rather, it requires waiting for information to arrive.

${ }^{22}$ Such a model may be relevant based on empirical evidence. Thus, Bowman and Moskowitz (2001, p. 775) suggest that one of the mistakes made by Merck when valuing its Project Gamma was the use of the BlackScholes formula, instead of taking into account that some biotechnology products follow a life cycle.
} 
expand or contract capacity, ${ }^{23}$ or R\&D investment opportunities that open up the option to enter new markets that evolve as the one described.

\footnotetext{
${ }^{23}$ In particular, Bollen (1998) examines these situations numerically. He shows that real options models that are based on a geometric brownian motion overvalue (undervalue) the option to expand (respectively, contract) a project.
} 


\section{REFERENCES}

Bar-Ilan, A. and William C. Strange (1996), "Investment Lags", American Economic Review, 86(3): 610-622.

Bernanke, B. S. (1983), “Irreversibility, Uncertainty and Cyclical Investment”, Quarterly Journal of Economics, 98(1): 85-106.

Bollen, N.P.B. (1999), "Real Options and Product Life Cycles", Management Science, 45(5): 670-684.

Bowman, E.H. and Gary T. Moskowitz (2001), "Real Options Analysis and Strategic Decision Making”, Organization Science, 12(6): 772-777.

Dixit, A. K. and Robert S. Pindyck (1994), "Investment under Uncertainty”, Princeton, NJ, Princeton University Press.

Ferderer, J.P. (1993), “The Impact of Uncertainty on Aggregate Investment Spending: An Empirical Analysis", Journal of Money, Credit and Banking, 25(1): 30-48.

Guiso, L. and Giuseppe Parigi (1999), "Investment and Demand Uncertainty”, Quarterly Journal of Economics, 114(1): 185-227.

Klette, T. and David de Meza (1986), “Is the Market Biased against Risky R\&D?”, Rand Journal of Economics, 17(1): 133-139.

Leahy, J.V. and Toni M. Whited (1996), “The Effect of Uncertainty on Investment: Some Stylized Facts", Journal of Money, Credit and Banking, 28(1): 64-83.

Mas-Colell, A., Michael D. Whinston and Jerry R. Green (1995), "Microeconomic Theory", Oxford, Oxford University Press.

McDonald, R.L. and Daniel Siegel, “The Value of Waiting to Invest”, Quarterly Journal of Economics, 101(4): 707-727. 
Pindyck, R.S. (1991), "Irreversibility, Uncertainty and Investment”, Journal of Economic Literature, 29(3): 1110-1148.

Roberts, K. and Martin L. Weitzman (1981), "Funding Criteria for Research, Development and Exploration Projects", Econometrica, 49(5): 1261-1288.

Samuel-Cahn, E. (1996), "Optimal Stopping with Random Horizon with Application to the Full-Information Best-Choice Problem with Random Freeze”, Journal of the American Statistical Association, 91(433): 357-364.

Tirole, J. (1988), “The Theory of Industrial Organization”, Cambridge, MA, MIT Press.

Trigeorgis, L. (1996), "Real Options: Managerial Flexibility and Strategy in Resource Allocation", Cambridge, MA, MIT Press.

Weeds, H. (2002), “Strategic Delay in a Real Options Model of R\&D Competition”, Review of Economic Studies, 69(3): 729-747. 


\section{APPENDIX}

Proof of Lemma 1: First we prove that, if immediate investment at the revealed maturity date $\tau$ is not preferred to delayed investment at $t^{\prime}>\tau$, then $N P V\left(t^{\prime}\right)=\int_{t^{\prime}}^{\infty} \pi e^{2 \alpha \tau} e^{-(\alpha+r) s} d s-K e^{-r t^{\prime}} \leq 0$, where $t^{\prime}$ and $\tau$ are arbitrary. Lastly, we show that immediate investment is profitable if and only if $\tau \geq t^{\max }$, which will complete the entire proof.

Regarding the first claim, note that $N P V(t)=\int_{t}^{\infty} \pi e^{2 \alpha \tau} e^{-(\alpha+r) s} d s-K e^{-r t}$ is a strictly quasiconvex function on $(-\infty,+\infty),{ }^{24} \quad$ with $\quad \lim _{t \rightarrow \infty} N P V(t)=0 \quad$ and $\hat{t} \equiv \underset{t}{\arg \min } N P V(t)=2 \tau+\frac{1}{\alpha} \log \left(\frac{\pi}{r K}\right)$. It suffices to show that the contrapositive of the claim is true, so assume that $N P V\left(t^{\prime}\right)>0$. Because of strict quasi-convexity and existence of the global minimum, we must have that either $\underset{t \in[\tau, \infty]}{\arg \max } N P V(t)=\infty$ or $\underset{t \in[\tau, \infty]}{\arg \max } N P V(t)=\tau$. In the first case, the fact that $\lim _{t \rightarrow \infty} N P V(t)=0$ would contradict the assumption that $N P V\left(t^{\prime}\right)>0$, by the definition of maximum. Hence, we must have that $\underset{t \in[\tau, \infty]}{\arg \max } N P V(t)=\tau$. But then $N P V(\tau)>N P V\left(t^{\prime}\right)$. This shows that the contrapositive is true.

As for the second claim, it clearly suffices to show that the entrant's payoff if it invested at $t^{\max }$ is zero, since $N P V(\tau)>0 \forall \tau>t^{\max } .{ }^{25}$ Let $t^{\max }>0$ and note that

${ }^{24}$ Formally, because $\frac{d N P V(t)}{d t}=0 \Rightarrow \frac{d^{2} N P V(t)}{d t^{2}}>0$.

${ }^{25}$ The proof is as follows. Let $\delta>0$. Then: 
$N P V\left(t^{\max }\right)=\int_{t_{\max }}^{\infty} \pi e^{2 \alpha t^{\max }} e^{-(\alpha+r) s} d s=\frac{\pi e^{(\alpha-r) t^{\max }}}{(\alpha+r)}-K e^{-r t^{\max }}=0 . \quad$ If $\quad t^{\max }=0, \quad$ then $N P V(0)=\frac{\pi-(\alpha+r) K}{(\alpha+r)}=0$. This completes the proof.

Proof of Lemma 2: We will first show that $V\left(t_{1}\right)$ is monotone decreasing if $t^{\max }=0$. In this case, the function becomes:

$$
\begin{aligned}
& V\left(t_{1}\right)=\int_{t_{\max }}^{t_{1}} f(\tau)\left(\int_{\tau}^{\infty} \Pi(s, \tau) e^{-r s} d s-K e^{-r \tau}\right) d \tau+\int_{t_{1}}^{\infty} f(\tau)\left(\int_{t_{1}}^{\infty} \Pi(s, \tau) e^{-r s} d s-K e^{-r t_{1}}\right) d \tau= \\
& \int_{t^{\max }}^{t_{1}} f(\tau)\left(\int_{\tau}^{\infty} \pi e^{2 \alpha \tau} e^{-(\alpha+r) s} d s-K e^{-r \tau}\right) d \tau+\int_{t_{1}}^{\infty} f(\tau)\left(\int_{t_{1}}^{\tau} \pi e^{(\alpha-r) s} d s+\int_{\tau}^{\infty} \pi e^{2 \alpha \tau} e^{-(\alpha+r) s} d s-K e^{-r t_{1}}\right) d \tau .
\end{aligned}
$$

Differentiating it with respect to $t_{1}$ and performing some algebraic manipulations yields:

$$
\frac{d V\left(t_{1}\right)}{d t_{1}} \equiv V^{\prime}\left(t_{1}\right)=\left(r K-\pi e^{\alpha t_{1}}\right) e^{-r t_{1}} \int_{t_{1}}^{\infty} f(\tau) d \tau
$$

We claim that $V^{\prime}\left(t_{1}\right)<0$ for $t_{1} \geq 0$. Otherwise, we would reach a contradiction:

$$
0 \leq\left(r K-\pi e^{\alpha t_{1}}\right) e^{-r t_{1}} \int_{t_{1}}^{\infty} f(\tau) d \tau \leq-\alpha K e^{-r t_{1}} \int_{t_{1}}^{\infty} f(\tau) d \tau<0
$$

since $t^{\max }=0$ if and only if $r K+\alpha K \leq \pi$. Given that Assumption 4 implies that $V\left(t_{1}\right)$ is bounded above, this shows that $V\left(t_{1}\right)$ attains a unique global maximum when $t^{\max }=0$.

To conclude the proof, let $t^{\max }>0$. Now note that $V\left(t_{1}\right)$ can be rewritten as follows:

$$
\begin{aligned}
N P V\left(t^{\max }+\delta\right)= & \frac{\pi e^{(\alpha-r)\left(t^{\max }+\delta\right)}}{\alpha+r}-K e^{-r\left(t^{\max }+\delta\right)}=e^{-r \delta}\left(\frac{\pi e^{(\alpha-r) t^{\max }} e^{\alpha \delta}}{\alpha+r}-K e^{-r t^{\max }}\right)= \\
& e^{-r \delta}\left(e^{\alpha \delta} K e^{-r t^{\max }}-K e^{-r t^{\max }}\right)>0,
\end{aligned}
$$

where the last equality follows from the definition of $t^{\max }$. 


$$
\begin{aligned}
V\left(t_{1}\right)= & \int_{t_{1}}^{\infty} f(\tau)\left(\int_{t_{1}}^{\infty} \Pi(s, \tau) e^{-r s} d s-K e^{-r t_{1}}\right) d \tau= \\
& \int_{t_{1}}^{\infty} f(\tau)\left(\int_{t_{1}}^{\tau} \pi e^{(\alpha-r) s} d s+\int_{\tau}^{\infty} \pi e^{2 \alpha \tau} e^{-(\alpha+r) s} d s-K e^{-r t_{1}}\right) d \tau
\end{aligned}
$$

By Assumption 4, this function is bounded above. Differentiating it with respect to $t_{1}$, solving the integrals, taking into account that $\lambda\left(t_{1}\right)=\frac{f\left(t_{1}\right)}{\int_{t_{1}}^{\infty} f(\tau) d \tau}$ and rearranging yields:

$$
\begin{aligned}
\frac{d V\left(t_{1}\right)}{d t_{1}} \equiv V^{\prime}\left(t_{1}\right) & =-\frac{\pi}{\alpha+r} e^{(\alpha-r) t_{1}} f\left(t_{1}\right)-\pi e^{(\alpha-r) t_{1}} \int_{t_{1}}^{\infty} f(\tau) d \tau+K e^{-r t_{1}}\left(f\left(t_{1}\right)+r \int_{t_{1}}^{\infty} f(\tau) d \tau\right) \\
& =\left(-\frac{\pi}{\alpha+r} e^{\alpha t_{1}} \lambda\left(t_{1}\right)-\pi e^{\alpha t_{1}}+K\left(r+\lambda\left(t_{1}\right)\right)\right) \int_{t_{1}}^{\infty} e^{-r t_{1}} f(\tau) d \tau
\end{aligned}
$$

If an interior maximum $t^{e}$ exists, it must be such that $V^{\prime}\left(t^{e}\right)=0$. Then multiplying through by $\frac{(\alpha+r)}{\pi \int_{t^{e}}^{\infty} e^{-r t^{e}} f(\tau) d \tau}$ and rearranging, the first-order condition can be written as follows: ${ }^{26}$

$$
e^{\alpha t^{e}}\left(1+\frac{\alpha}{r+\lambda\left(t^{e}\right)}\right)=\frac{K(\alpha+r)}{\pi}
$$

So if such $t^{e}$ exists, then it must be unique because the hazard-rate is non-increasing (the result follows from the intermediate value theorem and expression (A1)). Also, if $t^{e}$ exists, then $t^{e}<t^{\max }$. Otherwise, given that $t^{e}>0$, a contradiction would obtain using (A1) and the fact that $\frac{\alpha}{r+\lambda\left(t^{e}\right)}>0$ :

\footnotetext{
${ }^{26}$ If $\alpha=r$, then we should derive the functional form of $V\left(t_{1}\right)$ from scratch, since directly plugging in $\alpha=r$ implies that $V\left(t_{1}\right)$ is not well defined. Yet, straightforward calculations show that the same expression obtains for computing $t^{e}$.
} 


$$
t^{e} \geq t^{\max }=\frac{1}{\alpha} \log \left(\frac{K(\alpha+r)}{\pi}\right)=\frac{1}{\alpha} \log \left[e^{\alpha t^{e}}\left(1+\frac{\alpha}{r+\lambda\left(t^{e}\right)}\right)\right] \Rightarrow e^{\alpha t^{e}} \geq e^{\alpha t^{e}}\left(1+\frac{\alpha}{r+\lambda\left(t^{e}\right)}\right)
$$

Now we check that $V^{\prime \prime}\left(t^{e}\right)<0$, so that $t^{e}$ is indeed a global maximizer whenever it exists.

Since $V^{\prime}\left(t_{1}\right)=\left(-\frac{\pi}{\alpha+r} e^{\alpha t_{1}} \lambda\left(t_{1}\right)-\pi e^{\alpha t_{1}}+K\left(r+\lambda\left(t_{1}\right)\right)\right) \int_{t_{1}}^{\infty} e^{-r t_{1}} f(\tau) d \tau$, we have:

$$
\begin{aligned}
V^{\prime \prime}\left(t^{e}\right)= & -\left(f\left(t^{e}\right) e^{-r t^{e}}+\int_{t^{e}}^{\infty} r e^{-r t^{e}} f(\tau) d \tau\right)\left(-\frac{\pi}{\alpha+r} e^{\alpha t^{e}} \lambda\left(t^{e}\right)-\pi e^{\alpha t^{e}}+K\left(r+\lambda\left(t^{e}\right)\right)\right)+ \\
& \left(-\frac{\alpha \pi}{\alpha+r} e^{\alpha t^{e}} \lambda\left(t^{e}\right)-\frac{\pi}{\alpha+r} e^{\alpha t^{e}} \lambda^{\prime}\left(t^{e}\right)-\alpha \pi e^{\alpha t^{e}}+K \lambda^{\prime}\left(t^{e}\right)\right) \int_{t^{e}}^{\infty} e^{-r t^{e}} f(\tau) d \tau= \\
& {\left[-\frac{\alpha \pi}{\alpha+r} e^{\alpha t^{e}} \lambda\left(t^{e}\right)-\alpha \pi e^{\alpha t^{e}}+\lambda^{\prime}\left(t^{e}\right)\left(K-\frac{\pi}{\alpha+r} e^{\alpha t^{e}}\right)\right]_{t^{e}}^{\infty} e^{-r t^{e}} f(\tau) d \tau, }
\end{aligned}
$$

where we have made use of (A1). Given that $\lambda^{\prime}\left(t^{e}\right) \leq 0$ and $K>\frac{\pi}{\alpha+r} e^{\alpha t^{e}}$ because $t^{e}<t^{\max }$, it follows that $V^{\prime \prime}\left(t^{e}\right)<0$.

$t^{e}$ may not exist, though. Then we claim that $V(\cdot)$ must be monotone decreasing. To prove the claim, first note that $V\left(t^{\max }\right)>0$ by definition of $t^{\max }$. Second, it can be shown that $V(\cdot)$ is monotone decreasing on the set $\left[t^{\max }, \infty\right)$, since, on this region, we have:

$$
V\left(t_{1}\right)=\int_{t_{\max }}^{t_{1}} f(\tau)\left(\int_{\tau}^{\infty} \Pi(s, \tau) e^{-r s} d s-K e^{-r \tau}\right) d \tau+\int_{t_{1}}^{\infty} f(\tau)\left(\int_{t_{1}}^{\infty} \Pi(s, \tau) e^{-r s} d s-K e^{-r t_{1}}\right) d \tau
$$

Recall that we showed at the beginning of the proof that

$$
V^{\prime}\left(t_{1}\right)=\left(r K-\pi e^{\alpha t_{1}}\right) e^{-r t_{1}} \int_{t_{1}}^{\infty} f(\tau) d \tau
$$

Letting $t_{1}=t^{\max }+\delta$, where $\delta \geq 0$, we must have that $V^{\prime}\left(t^{\max }+\delta\right)<0$. Otherwise, the fact that $e^{\alpha \delta} \geq 1$ would lead us to the following contradiction: 


$$
0 \leq\left(r K-\pi e^{\alpha t^{\max }} e^{\alpha \delta}\right) e^{-r\left(t^{\max }+\delta\right)} \int_{t^{\max }+\delta}^{\infty} f(\tau) d \tau=\left(r K-r K e^{\alpha \delta}-\alpha K e^{\alpha \delta}\right) e^{-r\left(t^{\max }+\delta\right)} \int_{t^{\max }+\delta}^{\infty} f(\tau) d \tau<0 .
$$

Now it suffices to prove that $V(\cdot)$ is monotone decreasing on $\left[0, t^{\max }\right)$ if $t^{e}$ does not exist. We will do it by contradiction. Consider first the case in which $V(0) \geq V\left(t^{\max }\right)$. On the one hand, note that if $V(\cdot)$ is not monotonically decreasing on $\left[0, t^{\max }\right)$, then we cannot have $\frac{d V}{d t}(0)>0$. Otherwise, the fact that $V(0) \geq V\left(t^{\max }\right)$ implies that there must exist one critical point $\bar{t} \in\left(0, t^{\mathrm{max}}\right)$ at which $\frac{d V}{d t_{1}}(\bar{t})<0$, given the continuity and differentiability of $V(\cdot)$. Because $\frac{d V}{d t_{1}}(0)>0$, it follows from the continuous differentiability of $V(\cdot)$ that $\frac{d V}{d t_{1}}(\hat{t})=0$ for some $\hat{t} \in(0, \bar{t})$. The definition and uniqueness of $t^{e}$ imply that $\hat{t}=t^{e}$, which contradicts the non-existence of $t^{e}$. On the other hand, if $V(\cdot)$ were not monotonically decreasing and $\frac{d V}{d t_{1}}(0) \leq 0$, then exactly the same reasoning as before would lead to the existence of a critical point (a minimum in this case), which cannot be either. (The case in which $V(0)=V\left(t^{\max }\right)$ follows from the fact that the derivative of $V(\cdot)$ is clearly not equal to $0 \forall t_{1} \in\left[0, t^{\max }\right)$.) Hence, $V(\cdot)$ must be monotonically decreasing if $V(0) \geq V\left(t^{\max }\right)$. Therefore, a unique global maximum exists because $V(\cdot)$ is bounded. Now suppose that $V(\cdot)$ is not monotonically decreasing on $\left[0, t^{\max }\right)$, with $V(0)<V\left(t^{\max }\right)$. Then the continuity and differentiability of $V(\cdot)$ imply that there must be some $\bar{t} \in\left(0, t^{\max }\right)$ such that $\frac{d V}{d t_{1}}(\bar{t})>0$. Given that $\frac{d V}{d t_{1}}\left(t^{\max }\right)<0$, it follows that $\frac{d V}{d t_{1}}(\hat{t})=0$ for some 
$\hat{t} \in\left(\bar{t}, t^{\max }\right)$ (by the continuous differentiability of $\left.V(\cdot)\right)$. So we must have that such $\hat{t}$ is $t^{e}$, a contradiction. All this shows that we indeed have that $V(\cdot)$ must be monotone decreasing whenever $t^{e}$ does not exist, and thus $V(\cdot)$ attains a unique global maximum at date 0 .

Proof of Corollary 1: Algebraic manipulations yield:

$$
\pi e^{\alpha t^{e}}=\left(r+\lambda\left(t^{e}\right)\right) K-\lambda\left(t^{e}\right) \int_{t^{e}}^{\infty} \pi e^{\alpha\left(2 t^{e}-s\right)} e^{-r\left(s-t^{e}\right)} d s .
$$

Solving the integral and rearranging, we have:

$$
\pi e^{\alpha t^{e}}+\frac{\lambda\left(t^{e}\right) \pi e^{\alpha t^{e}}}{(\alpha+r)}=\left(r+\lambda\left(t^{e}\right)\right) K .
$$

Finally, multiplying both sides by $\frac{(\alpha+r)}{\pi\left(r+\lambda\left(t^{e}\right)\right)}$ yields expression (A1).

Proof of Proposition 1: Differentiate $G\left(t^{e}, \sigma^{2}\right)$ with respect to $t^{e}$ and $\sigma^{2}$ and rearrange so that:

$$
\begin{gathered}
\frac{d t^{e}}{d \sigma^{2}}\left(\sigma_{0}^{2}\right)=-\frac{e^{\alpha t_{0}^{e}}\left(\frac{-\alpha \lambda_{\sigma^{2}}\left(t_{0}^{e} \mid \sigma_{0}^{2}\right)}{\left(r+\lambda\left(t_{0}^{e} \mid \sigma_{0}^{2}\right)\right)^{2}}\right)}{\alpha e^{t_{0}^{e}}\left(\frac{\left(r+\lambda\left(t_{0}^{e} \mid \sigma_{0}^{2}\right)\right)^{2}+\alpha\left(r+\lambda\left(t_{0}^{e} \mid \sigma_{0}^{2}\right)\right)-\lambda_{t}\left(t_{0}^{e} \mid \sigma_{0}^{2}\right)}{\left(r+\lambda\left(t_{0}^{e} \mid \sigma_{0}^{2}\right)\right)^{2}}\right)}= \\
\frac{\lambda_{\sigma^{2}}\left(t_{0}^{e} \mid \sigma_{0}^{2}\right)}{\left(\left(r+\lambda\left(t_{0}^{e} \mid \sigma_{0}^{2}\right)\right)^{2}+\alpha\left(r+\lambda\left(t_{0}^{e} \mid \sigma_{0}^{2}\right)\right)-\lambda_{t}\left(t_{0}^{e} \mid \sigma_{0}^{2}\right)\right.} .
\end{gathered}
$$

Given that $\lambda_{t}\left(t_{0}^{e} \mid \sigma_{0}^{2}\right) \leq 0$ by assumption, we have that $\operatorname{sign}\left(\frac{d t^{e}}{d \sigma^{2}}\left(\sigma_{0}^{2}\right)\right)=\operatorname{sign}\left(\lambda_{\sigma^{2}}\left(t_{0}^{e} \mid \sigma_{0}^{2}\right)\right)$, which completes the proof.

Proof of Lemma 3: First note that $\pi^{*}\left(\sigma^{2}\right)$ is a well-defined function (and not a correspondence) by the uniqueness of $t^{e}$ for a given $\sigma^{2}$. Indeed, it is continuous by the continuity of $f\left(\cdot \mid \sigma^{2}\right) \forall \sigma^{2}$. Furthermore, if a mean-preserving spread is performed so that 
the variance infinitesimally rises from $\sigma_{0}^{2}$ to $\sigma_{1}^{2}$, then $f\left(0 \mid \sigma_{1}^{2}\right) \geq f\left(0 \mid \sigma_{0}^{2}\right)$, so $\pi^{*}\left(\sigma_{1}^{2}\right) \geq \pi^{*}\left(\sigma_{0}^{2}\right)$, which implies that $\pi^{*}\left(\sigma^{2}\right)$ is non-decreasing. Finally, note that $f\left(0 \mid \sigma^{2}\right)$ can be neither smaller than 0 nor larger than $\infty$, which, together with nondecreasingness of $\pi^{*}\left(\sigma^{2}\right)$, implies that the range of the function must be bounded by $[r K,(\alpha+r) K]$

Proof of Proposition 2: We proceed to prove the statement of the proposition for three different cases:

(i) If $\pi \geq \lim _{\sigma^{2} \rightarrow \infty} \pi^{*}\left(\sigma^{2}\right)$, then the monopolist's optimal time of entry is $t^{*}\left(\sigma^{2}\right)=0 \forall \sigma^{2},{ }^{27}$ so:

$$
V\left(\sigma_{0}^{2}\right)=\pi\left(\frac{1}{\alpha+r}+\frac{1}{\alpha-r}\right) \int_{0}^{\infty} e^{(\alpha-r) \tau} f\left(\tau \mid \sigma^{2}\right) d \tau-\left(\frac{\pi}{\alpha-r}+K\right)
$$

Hence, differentiating yields:

$$
V^{\prime}\left(\sigma_{0}^{2}\right) \equiv \frac{d V\left(\sigma_{0}^{2}\right)}{d \sigma^{2}}=\pi\left(\frac{1}{\alpha+r}+\frac{1}{\alpha-r}\right) \int_{0}^{\infty} e^{(\alpha-r) \tau} f_{\sigma^{2}}\left(\tau \mid \sigma^{2}\right) d \tau
$$

Integrating by parts, taking into account that $\int_{0}^{\infty} f_{\sigma^{2}}\left(\tau \mid \sigma_{o}^{2}\right) d \tau=0$ (which follows from differentiating both sides of the equation $\int_{0}^{\infty} f\left(\tau \mid \sigma_{0}^{2}\right) d \tau=1$ with respect to $\left.\sigma^{2}\right)$ and considering that $F_{\sigma^{2}}\left(\tau \mid \sigma_{0}^{2}\right)=\int_{0}^{\tau} f_{\sigma^{2}}\left(s \mid \sigma_{0}^{2}\right) d s$ when a mean-preserving spread is performed, we have:

\footnotetext{
${ }^{27}$ This follows from the facts that $\pi>\pi^{*}\left(\sigma^{2}\right) \forall \sigma^{2}$ if and only if the monopolist invests immediately for all $\sigma^{2}$.
} 


$$
\begin{aligned}
V^{\prime}\left(\sigma_{0}^{2}\right) & =\pi\left(\frac{1}{\alpha+r}+\frac{1}{\alpha-r}\right)\left(\left\{e^{(\alpha-r) \tau} \int_{0}^{\tau} f_{\sigma^{2}}\left(s \mid \sigma^{2}\right) d s\right]_{0}^{\infty}-\int_{0}^{\infty}(\alpha-r) e^{(\alpha-r) \tau} \int_{0}^{\tau} f_{\sigma^{2}}\left(s \mid \sigma^{2}\right) d s d \tau\right\} \\
& =-\left(\frac{2 \alpha \pi}{\alpha+r}\right) \int_{0}^{\infty} e^{(\alpha-r) \tau} F_{\sigma^{2}}\left(\tau \mid \sigma^{2}\right) d \tau
\end{aligned}
$$

Hence, taking into account that $t^{*}\left(\sigma_{0}^{2}\right)=t_{0}^{*}=0$ and $\int_{0}^{t_{0}^{*}} f_{\sigma^{2}}\left(\tau \mid \sigma_{0}^{2}\right) d \tau=0$ :

$$
\begin{aligned}
& V^{\prime}\left(\sigma_{0}^{2}\right) \geq 0 \text { iff } \int_{0}^{\infty} e^{(\alpha-r) \tau} F_{\sigma^{2}}\left(\tau \mid \sigma^{2}\right) d \tau \leq 0 \\
& \text { iff } 2\left(\alpha+\lambda\left(t_{0}^{*} \mid \sigma_{0}^{2}\right)\right) \int_{t_{0}^{*}}^{\infty} e^{(\alpha-r)\left(\tau-t_{0}^{*}\right)} F_{\sigma^{2}}\left(\tau \mid \sigma^{2}\right) d \tau \leq \int_{0}^{t_{0}^{*}} f_{\sigma^{2}}\left(\tau \mid \sigma_{0}^{2}\right) d \tau .
\end{aligned}
$$

This completes the proof when $\pi \geq \lim _{\sigma^{2} \rightarrow \infty} \pi^{*}\left(\sigma^{2}\right)$.

(ii) If $\pi \leq \lim _{\sigma^{2} \rightarrow 0} \pi^{*}\left(\sigma^{2}\right)$, then we have that $t^{*}\left(\sigma_{0}^{2}\right)=t^{e}\left(\sigma_{0}^{2}\right)>0 \forall \sigma_{0}^{2}$ by Lemma 3, so:

$$
\begin{array}{r}
V\left(\sigma^{2}\right)=\pi\left(\frac{1}{\alpha+r}+\frac{1}{\alpha-r}\right) \int_{t^{e}\left(\sigma^{2}\right)}^{\infty} e^{(\alpha-r) \tau} f\left(\tau \mid \sigma^{2}\right) d \tau- \\
\left(\frac{\pi e^{(\alpha-r) t^{e}\left(\sigma^{2}\right)}}{\alpha-r}+K e^{-r t^{e}\left(\sigma^{2}\right)}\right) \int_{t^{e}\left(\sigma^{2}\right)}^{\infty} f\left(\tau \mid \sigma^{2}\right) d \tau
\end{array}
$$

Given that $\frac{1}{\alpha} \log \left(\frac{r K}{\pi}\right) \leq t^{e}\left(\sigma^{2}\right) \leq \frac{1}{\alpha} \log \left(\frac{(\alpha+r) K}{\pi}\right) \forall \sigma^{2}$ (this trivially follows from Lemma 2), $t^{e}\left(\sigma^{2}\right)$ is a continuous function by the theorem of the maximum, with $\lim _{\sigma^{2} \rightarrow \infty} t^{e}\left(\sigma^{2}\right)<\infty$. In addition, we have that $t^{e}\left(\sigma^{2}\right)$ is differentiable on a local neighborhood of $\sigma_{0}^{2}$ by the implicit function theorem. By the envelope theorem, effects of $\sigma^{2}$ on $V(\cdot)$ via $t^{e}\left(\sigma^{2}\right)$ are of second-order, so letting $t^{e}\left(\sigma_{0}^{2}\right)=t_{0}^{e}$, we have:

$$
V^{\prime}\left(\sigma_{0}^{2}\right)=\pi\left(\frac{1}{\alpha+r}+\frac{1}{\alpha-r}\right) \int_{t_{0}^{e}}^{\infty} e^{(\alpha-r) \tau} f_{\sigma^{2}}\left(\tau \mid \sigma_{0}^{2}\right) d \tau-\left(\frac{\pi e^{(\alpha-r) t_{0}^{e}}}{\alpha-r}+K e^{-r t_{0}^{e}}\right)_{t_{0}^{e}}^{\infty} f_{\sigma^{2}}\left(\tau \mid \sigma_{0}^{2}\right) d \tau
$$


Integrating by parts and recalling that $\int_{0}^{\infty} f_{\sigma^{2}}\left(\tau \mid \sigma_{0}^{2}\right) d \tau=0 \quad$ and $F_{\sigma^{2}}\left(\tau \mid \sigma_{0}^{2}\right)=\int_{0}^{\tau} f_{\sigma^{2}}\left(s \mid \sigma_{0}^{2}\right) d s$ when a mean-preserving spread is performed yields:

$$
\begin{aligned}
& V^{\prime}\left(\sigma_{0}^{2}\right)=-\left(\frac{\pi e^{(\alpha-r) t_{0}^{e}}}{\alpha-r}+K e^{-r t_{0}^{e}} \int_{t_{0}^{e}}^{\infty} f_{\sigma^{2}}\left(\tau \mid \sigma_{0}^{2}\right) d \tau+\right. \\
& \pi\left(\frac{1}{\alpha+r}+\frac{1}{\alpha-r}\right)\left\{\left[e^{(\alpha-r) \tau} \int_{0}^{\tau} f_{\sigma^{2}}\left(s \mid \sigma_{0}^{2}\right) d s\right]_{t_{0}^{e}}^{\infty}-\int_{t_{0}^{e}}^{\infty}(\alpha-r) e^{(\alpha-r) \tau} \int_{0}^{\tau} f_{\sigma^{2}}\left(s \mid \sigma_{0}^{2}\right) d s d \tau\right\}= \\
& \left(\frac{\pi e^{(\alpha-r) t_{0}^{e}}}{\alpha+r}+\frac{\pi e^{(\alpha-r) t_{0}^{e}}}{\alpha-r}-\frac{\pi e^{(\alpha-r) t_{0}^{e}}}{\alpha-r}-K e^{-r t_{0}^{e}} \int_{t_{0}^{e}}^{\infty} f_{\sigma^{2}}\left(\tau \mid \sigma_{0}^{2}\right) d \tau-\left(\frac{2 \alpha \pi}{\alpha+r}\right) \int_{t_{0}^{e}}^{\infty} e^{(\alpha-r) \tau} F_{\sigma^{2}}\left(\tau \mid \sigma^{2}\right) d \tau=\right. \\
& \left(\frac{\pi e^{(\alpha-r) t_{0}^{e}}}{\alpha+r}-K e^{-r t_{0}^{e}}\right) \int_{t_{0}^{e}}^{\infty} f_{\sigma^{2}}\left(\tau \mid \sigma^{2}\right) d \tau-\left(\frac{2 \alpha \pi}{\alpha+r}\right) \int_{t_{0}^{e}}^{\infty} e^{(\alpha-r) \tau} F_{\sigma^{2}}\left(\tau \mid \sigma^{2}\right) d \tau
\end{aligned}
$$

Hence, we have that

$$
\begin{gathered}
\mathrm{IO}^{\prime}\left(\sigma_{0}^{2}\right) \geq 0 \text { iff }\left(\frac{2 \alpha \pi}{\alpha+r}\right) \int_{t_{0}^{e}}^{\infty} e^{(\alpha-r) \tau} F_{\sigma^{2}}\left(\tau \mid \sigma^{2}\right) d \tau \leq\left(-\frac{\alpha \pi e^{(\alpha-r) t_{0}^{e}}}{(\alpha+r)\left(\alpha+\lambda\left(t_{0}^{e} \mid \sigma_{0}^{2}\right)\right)}\right) \int_{t_{0}^{e}}^{\infty} f_{\sigma^{2}}\left(\tau \mid \sigma_{0}^{2}\right) d \tau \\
\quad \text { iff } 2\left(\alpha+\lambda\left(t_{0}^{e} \mid \sigma_{0}^{2}\right)\right) \int_{t_{0}^{e}}^{\infty} e^{(\alpha-r)\left(\tau-t_{0}^{e}\right)} F_{\sigma^{2}}\left(\tau \mid \sigma^{2}\right) d \tau \leq-\int_{t_{0}^{e}}^{\infty} f_{\sigma^{2}}\left(\tau \mid \sigma_{0}^{2}\right) d \tau \\
\quad \text { iff } 2\left(\alpha+\lambda\left(t_{0}^{e} \mid \sigma_{0}^{2}\right)\right) \int_{t_{0}^{e}}^{\infty} e^{(\alpha-r)\left(\tau-t_{0}^{e}\right)} F_{\sigma^{2}}\left(\tau \mid \sigma^{2}\right) d \tau \leq \int_{0}^{t_{0}^{e}} f_{\sigma^{2}}\left(\tau \mid \sigma_{0}^{2}\right) d \tau,
\end{gathered}
$$

where first we have used the first order condition of the optimization program and finally we have used the fact that $\int_{0}^{\infty} f_{\sigma^{2}}\left(\tau \mid \sigma_{0}^{2}\right) d \tau=0$.

(iii) If $\lim _{\sigma^{2} \rightarrow \infty} \pi^{*}\left(\sigma^{2}\right)>\pi \geq \lim _{\sigma^{2} \rightarrow 0} \pi^{*}\left(\sigma^{2}\right)$, define $\Sigma(\pi) \equiv\left\{\sigma^{2} \in[0, \infty): \pi\left(\sigma^{2}\right)=\pi\right\}$. Since $\pi^{*}\left(\sigma^{2}\right)$ is continuous by Lemma 3, then $\Sigma(\pi)$ must be convex (if not a singleton), so the following facts clearly hold: (1) case (i) applies $\forall \sigma^{2}<\inf \Sigma(\pi)$; (2) case (ii) applies $\forall \sigma^{2} \geq \inf \Sigma(\pi)$; and (3) $t^{e}\left(\sigma_{0}^{2}\right) \downarrow 0$ as $\pi \uparrow \pi^{*}\left(\sigma_{0}^{2}\right)$, so $t^{*}\left(\sigma^{2}\right)$ is a 
continuous function (since $\pi^{*}\left(\sigma^{2}\right)$ is continuous). This shows that, although $V\left(\sigma^{2}\right)$ need not be differentiable at $\inf \Sigma(\pi)$, it is clearly continuous and non-decreasing under the conditions stated in the proposition. 